\title{
TO STUDY THE USE OF HYDROXYCHLOROQUINE IN SMALL DOSES IN REGRESSION OF DIABETIC NEPHROPATHY IN PATIENTS OF TYPE II DIABETES MELLITUS
}

\author{
Jitendra Singh Kushwaha1, Shri Krishna Gautam², Harshit Khare ${ }^{3}$ \\ 1 Professor, Department of Medicine, GSVM Medical College, Kanpur, U. P. \\ ${ }^{2}$ Assistant Professor, Department of Medicine, GSVM Medical College, Kanpur, U. P. \\ 3Junior Resident, Department of Medicine, GSVM Medical College, Kanpur, U. P.
}

\begin{abstract}
\section{BACKGROUND}

The outcomes with the armamentarium of drugs targeting the progression of Nephropathy in Type II Diabetic patients are not promising with most of the patients needing haemodialysis ultimately. Hydroxychloroquine (HCQS), an anti-malarial used mainly for treatment in Rheumatoid arthritis, Psoriasis and Lupus nephritis had been recently approved by Drug Controller General of India, in 2015, as an anti-Diabetic drug. With recent studies, HCQS was found to decrease proteinuria and retarded the progression of nephropathy in Lupus nephritis patients. In few of the studies, Hydroxychloroquine also proved to have favourable effects on Lipid profile of subjects. We decided to investigate Hydroxychloroquine in Diabetic Nephropathy patients, proposing that it could retard the progression of Nephropathy in Type II Diabetics, just as it proved beneficial and disease modifying in Lupus Nephritis patients.
\end{abstract}

\section{MATERIALS AND METHODS}

This study was non-randomised controlled trial. A total of 67 patients with Type II Diabetes Mellitus (in the age group of 30 - 80 yrs. with clinical evidence of Diabetic Kidney Disease [DKD], HbA1c $\geq 6.5$ ) were selected among admitted patients of the KPS PG Institute of Medicine at LLR and Associated Hospital, GSVM Medical College, Kanpur and alternatively divided into two groups with one group taking Hydroxychloroquine $(100 \mathrm{mg}$ b.i.d.) in addition to their regular medications. Both groups were then looked for any significant changes in their renal function after 3 months.

\section{RESULTS}

In our study with S. creatinine there was decline of 19.99\% from baseline T0 to T3 in Hydroxychloroquine group, while it increased from T0 to T3 in Non-Hydroxychloroquine group by around $27.56 \%$, both of which were significant (P<.001). Similarly, eGFR was found to be rising by $27.9 \%$ in the Hydroxychloroquine group and decreased by $25.11 \%$ in the Non-Hydroxychloroquine group, again which was statistically significant $(\mathrm{P}<.001)$. Also, Urinary ACR decreased by $26.59 \%$ in the Hydroxychloroquine group, but continued to increase by $19.63 \%$ in the Non-Hydroxychloroquine group, both of which were statistically significant $(\mathrm{P}<.001)$.

\section{CONCLUSION}

While eGFR progressively declined in the Non-Hydroxychloroquine group, an improvement in eGFR was observed in the Hydroxychloroquine group. Therefore, we concluded that HCQS can be an option in patients of early stages of DKD for regression of Diabetic Nephropathy.

\section{KEYWORDS}

Diabetic Nephropathy, Hydroxychloroquine.

HOW TO CITE THIS ARTICLE: Kushwaha JS, Gautam SK, Khare H. To study the use of hydroxychloroquine in small doses in regression of diabetic nephropathy in patients of type II diabetes mellitus. J. Evolution Med. Dent. Sci. 2018;7(03):346-350, DOI: $10.14260 /$ jemds/2018/77

\section{BACKGROUND}

Towards the end of 20th century, due to decreased mortalities from Ischaemic Heart Disease and more aging population, incidence of diseases, unnoticeable till then increased to catch attention of the scientific community. Diabetic nephropathy/ Diabetic kidney disease was one of them. This resulted in continuous attempts to find a way around this puzzle.

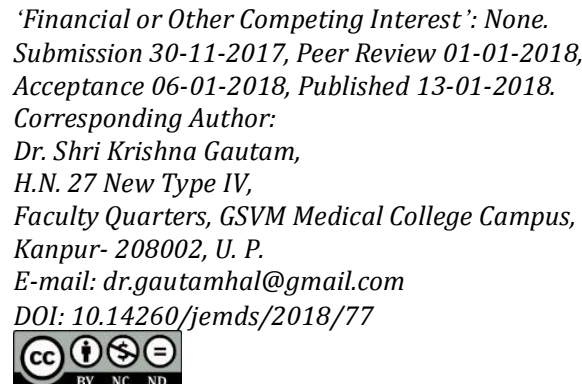

Lately, Angiotensin Converting Enzyme inhibitors (ACEi) like Lisinopril and Angiotensin Receptor Blockers (ARB) like Valsartan, Candesartan, Losartan, some Calcium Channel Blockers (CCB) like Nisoldipine, Lacidipine, Statins and Spironolactone were all investigated as drugs targeting the progression of Nephropathy in Type II Diabetic patients and yet the outcomes were not promising with most of the patients needing haemodialysis ultimately. As new mechanisms underlying the pathogenesis of Diabetic Kidney Disease are being discovered namely proteinuria, role of dyslipidaemia and chronic hyperglycaemia, we conducted a study with the objective to establish the effectiveness of Hydroxychloroquine in regression of Diabetic Nephropathy.1,2,3

\section{MATERIALS AND METHODS}

This study was non-randomised controlled trial. Sixty seven patients of Type II Diabetes mellitus, irrespective of sex, in the age group of 30 - 80 yrs. with clinical evidence of Diabetic 
Kidney Disease [DKD] were selected from set of patients admitted and willing to give consent in the KPS PG Institute of Medicine at LLR and Associated Hospital, GSVM Medical College, Kanpur from December 2015 to May 2017.

\section{Diabetic Kidney Disease was defined as-}

Either having Class II - V of kidney disease on histopathology in renal biopsy or proteinuria $\geq 0.5$ g per 24 hours or $3+$ proteinuria without UTI; or

One of the following features also attributable to DKD and present on two or more visits performed at least three months apart:

Proteinuria $\geq 2+$, creatinine clearance $\leq 90 \mathrm{~mL} / \mathrm{min}, \geq 10$ RBCs or WBCs per high-power field (HPF) or $\geq 3$ granular or cellular casts per HPF or microalbuminuria.

Microalbuminuria defined as urinary albumin excretion of $30-299 \mathrm{mg} / 24$ hours in a 24-hour urinary collection or 20 $199 \mathrm{ug} / \mathrm{min}$ in a timed urine collection or $30-299 \mathrm{ug} / \mathrm{mg}$ creatinine in a spot urine collection on at least two occasions.

Creatinine was measured at the laboratories of the participating institutions by the modified method of Jaffe in which creatinine combines with picrate in an alkaline solution to form a creatinine-picrate complex.

\section{Study Period}

December 2015 to May 2017.

\section{Place of Study}

Inpatient and Outpatients of KPS Postgraduate Department of Medicine at LLR and Associated Hospital, GSVM Medical College, Kanpur.

\section{Inclusion Criteria}

Patients with history of Diabetes Mellitus type II for at least 5 yrs. with Diabetic Kidney Disease [DKD] defined as urinary albumin excretion of $30-299 \mathrm{mg} / 24$ hours in a 24-hour urinary collection or 20 - 199 microgram/ min in a timed urine collection or $30-299$ microgram/mg creatinine in a spot urine collection on at least two occasions; or eGFR $<90$ $\mathrm{mL} / \mathrm{min} / 1.73 \mathrm{~m}^{2}$, with $\mathrm{HbA} 1 \mathrm{c}>6.5$, age between 30 to 80 yrs. with/ without documented medical renal disease on abdominal ultrasonography.

\section{Exclusion Criteria}

- DKD patients on maintenance haemodialysis.

- DKD patients with symptoms/ signs of uraemia.

- DKD with history of Congestive Heart Failure (CHF) or currently with symptoms and signs of CHF.

- DKD with history of coronary artery disease.

- DKD with proliferative retinopathy or non-proliferative retinopathy with age $>60$ yrs.

- DKD patients who had ongoing urinary tract infection with pus cells in urine.

After taking complete informed consent in native language, HCQS was started at dose of $100 \mathrm{mg}$ given as twice daily regimen along with other regular medication and short acting insulin. All patients were assessed at Hospital initially and then after 3 months further. Dosage of drugs for the treatment of diabetes and hypertension was adjusted to maintain good glycaemic and blood pressure control from then onwards. Antihypertensive drugs included amlodipine, nifedipine and moxonidine.

\section{Investigations}

At start of study and following 3 months into study each patient was investigated for the following-

- HbA1c.

- Serum lipid profile.

- Blood sugar- Fasting and Post prandial.

- Serum creatinine level.

- Serum urea.

- Liver Function Test.

- USG whole abdomen.

- Urine routine and microscopic examination.

- Urine albumin creatinine ratio.

- $\quad$ ECG and 2D-Echo.

- $\mathrm{CT}$ abdomen if required.

At each visit, body weight and blood pressure were measured and fasting blood samples were collected for the measurement of above investigations.

Glomerular filtration rate (GFR) was measured using Modification of Diet in Renal Disease (MDRD) Study formula: eGFR $=186 \times$ (Creatinine in mg/ dL) $-1.154 \times$ (Age) $-0.203 \times$ ( 0.742 if female) $\times$ (1.210 if black).

\section{Grouping of Cases}

Patients after screening, were selected for the study and were alternatively grouped as follows-

- Group I- (Hydroxychloroquine/ HCQS group/ cases)Patients with established DKD, who gave consent and were willing to take HCQS in the dosage of $200 \mathrm{mg}$ daily for at least 3 months in addition to their other medications.

- Group 2- (Non-Hydroxychloroquine/ Non-HCQS group/ controls)- Patients with established DKD who were not given HCQS for the same duration of time in addition to their regular medication.

Each patient had a baseline or enrolment visit (T0) followed by a 3-month visit (T3). Time of screening (Ts) was defined as the time when each patient met for ACR criteria. Each visit included interview, physical examination and laboratory tests.

Additional clinical information covering the period between scheduled visits as well as data for missed study visits were obtained by review of all available medical records.

None of the patients had episodes of hypoglycaemia since the diagnosis of Type II Diabetes upto present and during the period of the study.

All patients were on short-acting insulin with dosage modified according to daily insulin need and changes in insulin requirement with changing renal function. They had been on short-acting insulin for at least 3 months prior to the study.

During the study, all were recommended and advised to take a diet consisting of $30 \mathrm{kcal}$ per $\mathrm{kg}$ ideal body weight, $50 \%-55 \%$ carbohydrate, $30 \%$ fat, $15 \%-20 \%$ protein and $300 \mathrm{mg}$ cholesterol, which was reinforced during the period of the study in both the groups. 
Patients in both the groups were taking statins as hypolipidaemic drugs during the period of study in low-risk dose. Four patients withdrew from the study after 3 months, three could not attend the frequent follow-up due to personal reasons and another emigrated.

None of the patients in either group went for haemodialysis during or before the period of study.
Data was compiled using numbers version 4.3 .1 and analysed using IBM SPSS Statistics version 23 to compare baseline characteristics of the groups, and independent t-test was done. To find out variation in variables in both the study groups during the study period, paired t-test was applied. The clinical characteristics of the 67 patients who completed the 3 months study are summarised in Table 1 .

\section{Statistical Methods}

\begin{tabular}{|c|c|c|c|c|c|c|}
\hline \multicolumn{7}{|c|}{ Group Statistics } \\
\hline & Code & $\mathbf{N}$ & Mean & Std. Deviation & Std. Error Mean & P value \\
\hline \multirow[t]{2}{*}{ HbA1c T0 } & 1 & 29 & 9.26 & 2.196 & 0.408 & .091 \\
\hline & 2 & 36 & 9.52 & 1.549 & 0.258 & \\
\hline \multirow[t]{2}{*}{ S. crea. T0 } & 1 & 31 & 2.01 & 0.878 & 0.158 & .701 \\
\hline & 2 & 36 & 1.56 & 1.000 & 0.167 & \\
\hline \multirow[t]{2}{*}{ eGFR. T0 } & 1 & 31 & 39.95 & 23.764 & 4.268 & .808 \\
\hline & 2 & 36 & 55.99 & 23.867 & 3.978 & \\
\hline \multirow[t]{2}{*}{ BS-F. T0 } & 1 & 29 & 214.73 & 84.904 & 15.766 & .451 \\
\hline & 2 & 36 & 227.13 & 44.357 & 7.393 & \\
\hline \multirow{2}{*}{ BS-PP. T0 } & 1 & 28 & 320.96 & 104.283 & 19.708 & .944 \\
\hline & 2 & 36 & 319.49 & 63.225 & 10.537 & \\
\hline \multirow{2}{*}{ U. ACR. T0 } & 1 & 31 & 1210.206 & 535.0952 & 96.1059 & .085 \\
\hline & 2 & 36 & 929.414 & 460.8613 & 76.8102 & \\
\hline \multirow[t]{2}{*}{ CHO. T0 } & 1 & 18 & 177.87 & 46.623 & 10.989 & 0.840 \\
\hline & 2 & 36 & 175.89 & 26.082 & 4.347 & \\
\hline \multirow[t]{2}{*}{ TG. T0 } & 1 & 18 & 183.96 & 109.797 & 25.879 & .379 \\
\hline & 2 & 36 & 202.91 & 29.833 & 4.972 & \\
\hline \multirow[t]{2}{*}{ HDL. T0 } & 1 & 18 & 57.61 & 20.191 & 4.759 & .292 \\
\hline & 2 & 36 & 41 & 7.724 & 1.287 & \\
\hline \multirow[t]{2}{*}{ LDL. T0 } & 1 & 18 & 81.67 & 42.763 & 10.079 & .115 \\
\hline & 2 & 36 & 93.40 & 15.640 & 2.607 & \\
\hline & & & Baseli & istics & & \\
\hline
\end{tabular}

\section{RESULTS}

There was no significant difference between Hydroxychloroquine group and Non-Hydroxychloroquine group patients in any of the clinical characteristics at baseline (Table 1). With serum creatinine there was decline of $19.99 \%$ from baseline T0 to T3 in Hydroxychloroquine group, which was significant. However, serum creatinine increased from T0 to T3 in Non-Hydroxychloroquine group by around $27.56 \%$, which was also significant (Table 2, Figure 1). Estimated glomerular filtration rate was found to be rising by $27.9 \%$ in the Hydroxychloroquine group from baseline T0 to T3 and decreased by $25.11 \%$ in the Non-Hydroxychloroquine group from baseline T0 to T3, again which was statistically significant (Table 3, Figure 2).

Urinary Albumin-Creatinine ratio decreased by $26.59 \%$ in the Hydroxychloroquine from baseline T0 to T3 group and continued to increase by $19.63 \%$ in the NonHydroxychloroquine group from baseline T0 to T3, both of which were statistically significant (Table 4, Figure 3 ).

\begin{tabular}{|c|c|c|}
\hline Groups/Time & $\begin{array}{c}\text { S. creatinine T0 } \\
\text { Mean } \pm \text { SD }\end{array}$ & $\begin{array}{c}\text { S. creatinine T3 } \\
\text { Mean } \pm \text { SD }\end{array}$ \\
\hline Hydroxychloroquine & $2.01 \pm 0.805$ & $1.61 \pm 0.703$ \\
\hline $\begin{array}{c}\text { Non- } \\
\text { Hydroxychloroquine }\end{array}$ & $1.56 \pm 0.788$ & $1.99 \pm 0.866$ \\
\hline \multicolumn{2}{|c|}{ Table 2. Serum Creatinine Mean Variation } \\
\hline
\end{tabular}

For Hydroxychloroquine group, s. creatinine mean value was 2.01 initially at start of study (T0) with SD of \pm .805 and after 3 months (T3) S. creatinine mean value was 1.61 with $\mathrm{SD} \pm .703$. Paired ' $\mathrm{t}$ ' test was applied, and $\mathrm{p}$ value was $<0.001$ indicating the decline in S. creatinine was highly significant.

For the Non-Hydroxychloroquine group, S. creatinine mean value was 1.56 initially at start of study (T0) with SD of \pm 0.788 and after 3 months (T3) S. creatinine mean value was 1.99 with $S D \pm 0.866$. Paired ' $t$ ' test was applied, and $p$ value was $<0.001$ indicating the increase in S. creatinine was highly significant in the Non-HCQS group (Table 2, Figure 1).

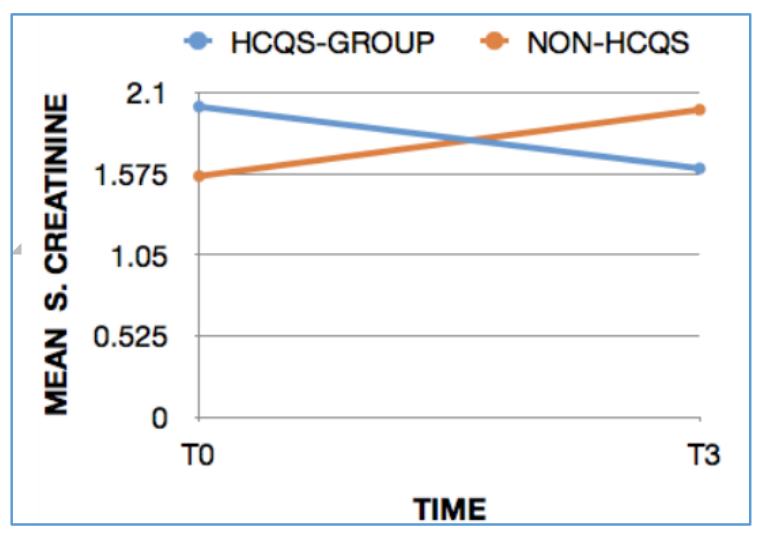

Figure 1. S. Creatinine Variation 


\begin{tabular}{|c|c|c|}
\hline Groups/ Time & $\begin{array}{c}\text { eGFR T0 } \\
\text { Mean } \pm \text { SD }\end{array}$ & $\begin{array}{c}\text { eGFR T3 } \\
\text { Mean } \pm \text { SD }\end{array}$ \\
\hline Hydroxychloroquine & $39.95 \pm 19.970$ & $51.10 \pm 23.169$ \\
\hline Non-Hydroxychloroquine & $55.99 \pm 21.241$ & $41.93 \pm 19.112$ \\
\hline \multicolumn{3}{|c|}{ Table 3. eGFR Mean Variation }
\end{tabular}

For HCQS group, eGFR mean value was 39.95 initially at start of study (T0) with SD of \pm 19.970 and after 3 months (T3), eGFR mean value was 51.10 with $\mathrm{SD} \pm 23.169$. Paired ' $\mathrm{t}$ ' test was applied, and $\mathrm{p}$ value was $<0.001$ indicating the increase in eGFR was highly significant.

For the Non-HCQS group, eGFR mean value was 55.99 initially at start of study (T0) with SD of \pm 21.241 and after 3 months (T3), eGFR mean value was 41.93 with SD \pm 19.112 . Paired ' $\mathrm{t}$ ' test was applied, and $\mathrm{p}$ value was $<0.001$ indicating the decline in eGFR, was highly significant in the Non-HCQS group (Table 3, Figure 2).

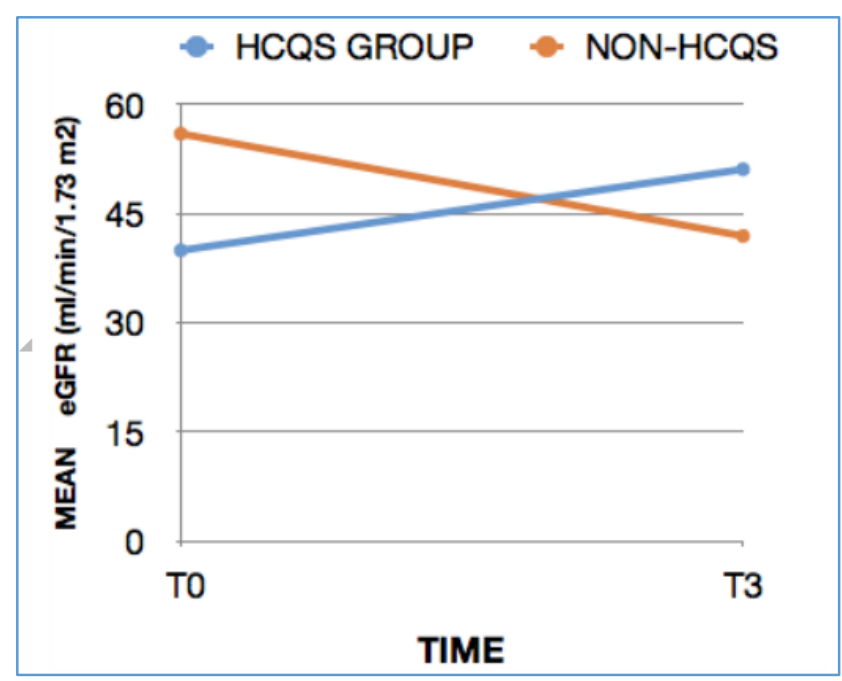

Figure 2. eGFR Mean Variation

\begin{tabular}{|c|c|c|}
\hline Groups/ Time & U. ACR T0 & U. ACR T3 \\
& Mean \pm SD & Mean \pm SD \\
\hline \multirow{2}{*}{ Hydroxychloroquine } & $1210.206 \pm$ & $888.299 \pm$ \\
& 394.72 & 245.3529 \\
\hline \multirow{2}{*}{ Non-Hydroxychloroquine } & $929.414 \pm$ & $1111.931 \pm$ \\
& 266.6158 & 500.78 \\
\hline \multicolumn{2}{|c|}{ Table 4. U. ACR Mean Variation } \\
\hline
\end{tabular}

For Hydroxychloroquine group, U. ACR mean value was 1210.206 initially at start of study (T0) with SD of \pm 394.72 and after 3 months (T3), U. ACR mean value was 888.299 with SD \pm 245.3529 . Paired ' $t$ ' test was applied, and $p$ value was $<0.001$ indicating the decline in U. ACR was highly significant.

For the Non-Hydroxychloroquine group, U. ACR mean value was 929.414 initially at start of study (T0) with SD of \pm 266.6158 and after 3 months (T3) U. ACR mean value was 1111.931 with SD \pm 500.78 . Paired ' $t$ ' test was applied, and $p$ value was $<0.001$ indicating the increase in U. ACR, was highly significant in the Non-Hydroxychloroquine group (Table 4, Figure 3).

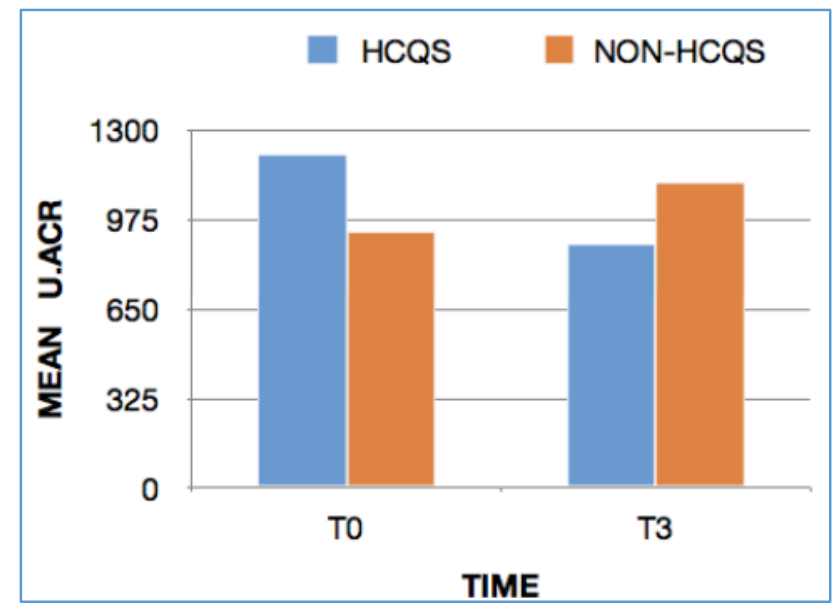

Figure 3. U. ACR Mean Variation

\section{DISCUSSION}

We aimed to find if there was any role of Hydroxychloroquine in retarding or even better reversing the Diabetes associated nephropathy. This was effected by screening the Type II Diabetic patients with history, symptoms and signs of Nephropathy from the general population of Type II Diabetics using pre-decided Inclusion and Exclusion criteria (Discussed in Material and Methods).

They were then alternatively divided into two Groups with one group taking Hydroxychloroquine in dosage of 100 mg twice daily along with their rest of the medication.

Rest of the Medication included short-acting Insulin, Amlodipine/ Nifedipine, Atorvastatin in low-risk dosage, Torsemide, Vit D preparations, Calcium preparations and Angiotensin-Converting Enzyme Inhibitors (Ramipril).

The other group (Non-Hydroxychloroquine group) continued with rest of the medications based on short-acting insulin without Hydroxychloroquine.

Both groups were followed for next 3 months and serial changes in their S. creatinine, eGFR, Urine ACR, HbA1c, BS-F, PP, LDL, HDL and TG were noted.

The total number of patients on ramipril in both the groups were 16/ 31 in the Hydroxychloroquine group and 22/ 36 in the non-Hydroxychloroquine group respectively based on whether their eGFR permitted use of ramipril or not.

Total 4 patients were withdrawn from ramipril during the period of study ( 2 in each group) as their eGFR declined.

In our study with $\mathrm{S}$. creatinine there was decline of 19.99\% from baseline T0 to T3 in Hydroxychloroquine group, which was significant. However, S. creatinine increased from T0 to T3 in Non-Hydroxychloroquine group by around $27.56 \%$, which was also significant (Table 3 ).

Although, proteinuria is a good prognostic index of progressive renal disease in early stages of nephropathy, urinary protein excretion rate may fall when the GFR becomes significantly reduced so that it becomes less reliable as an index of progressive renal disease. So we decided to measure U. ACR for baseline assessment and later on after 3 months followup.

U. ACR decreased by $26.59 \%$ in the Hydroxychloroquine group from baseline T0 to T3 and continued to increase by $19.63 \%$ in the Non-Hydroxychloroquine group from baseline T0 to T3, both of which were statistically significant. 
eGFR was found to be rising by $27.9 \%$ in the Hydroxychloroquine group from baseline T0 to T3 and decreased by $25.11 \%$ in the Non-Hydroxychloroquine group from baseline $\mathrm{T} 0$ to $\mathrm{T} 3$, again which was statistically significant.

This has been supported by previous studies too, whereby targeting microalbuminuria many drugs have went into clinical trials with the view of retarding Nephropathy in CKD patients as well as in Diabetic patients. ${ }^{4}$

To support this, we had CALM Study by Mogensen et $\mathrm{al}^{5}$ where they assessed and compared the effects of candesartan or lisinopril or both on blood pressure and urinary albumin excretion in patients with microalbuminuria, hypertension and type 2 diabetes.

\section{CONCLUSION}

While eGFR progressively declined in the NonHydroxychloroquine group, an improvement in eGFR was observed in the Hydroxychloroquine group.

The changes in serum creatinine were also in keeping with an attenuating effect of HCQS on the deterioration of glomerular function in these patients.

A beneficial effect of Hydroxychloroquine on GFR in Lupus Nephritis patients has been previously reported in LUMINA study. 6,7

The current therapy for chronic proteinuric nephropathies is angiotensin-converting enzyme inhibitors (ACEi), which slows but may not halt the progression of disease and which may be not effective to the same degree in all patients.

With more and more mechanisms being unveiled every year with ongoing research in the field of Diabetes and Diabetic nephropathy, we have multiple targets to explore further attempting to solve the puzzle of retarding progression of DKD. Will HCQS be the key to unlock that puzzle? Of that we are not sure, but it certainly holds the potential.
Though presented study here was limited by relatively small sample size and short duration of treatment, we conclude that HCQS can be an option in patients of early stages of DKD to retard the progression of disease.

\section{REFERENCES}

[1] Winter EM, van der Meer AS, Eustatia-Rutten C, et al Hydroxychloroquine as a glucose lowering drug. BMJ Case Reports 2011;2011: pii. bcr0620114393.

[2] Pareek A, Chandurkar N, Thomas N, et al. Efficacy and safety of hydroxychloroquine in the treatment of type 2 diabetes mellitus: a double blind, randomized comparison with pioglitazone. Curr Med Res Opin 2014;30(7):1257-66.

[3] Pareek A. Ipca gets first approval for HCQ in diabetes. SCRIP Intelligence 2015.

[4] Cooper ME. Pathogenesis, prevention and treatment of diabetic nephropathy. Lancet 1998;352(9123):213-9.

[5] Mogensen CE, Neldam S, Tikkanen I, et al. Randomised controlled trial of dual blockade of renin-angiotensin system in patients with hypertension, microalbuminuria and non-insulin dependent diabetes: The Candesartan and Lisinopril Microalbuminuria (CALM) study. BM] 2000;321(7274):1440-4.

[6] Griffin B, Lightstone L. Renoprotective strategies in lupus nephritis: beyond immunosuppression. Lupus 2013;22(12):1267-73.

[7] Sisó A, Ramos-Casals M, Bové A, et al. Previous antimalarial therapy in patients diagnosed with lupus nephritis: influence onoutcomes and survival. Lupus 2008;17(4):281-8. 